\title{
Comportamento de Escolares Estratificados pelo Peso ao Nascer e Idade Gestacional $^{1}$
}

\author{
Sabrina Kerr Bullamah Correia ${ }^{2}$ \\ Adriana Martins Saur \\ Sonia Regina Loureiro \\ Universidade de São Paulo
}

\begin{abstract}
RESUMO - O peso e a idade gestacional são critérios relevantes para avaliação das condições de nascimento das crianças. O objetivo do presente estudo foi caracterizar e comparar o desempenho comportamental de uma coorte de crianças estratificada pelo peso ao nascer e pelo peso ao nascer em relação à idade gestacional. Avaliaram-se, aos 10/11 anos, 677 crianças por meio do Questionário de Capacidades e Dificuldades. Verificou-se, com significância estatística, os seguintes resultados: o grupo Baixo Peso apresentou mais sintomas emocionais que o grupo Peso Normal; o grupo Muito Baixo Peso mostrou mais hiperatividade em comparação aos outros; o grupo Pequeno para a Idade Gestacional apresentou mais dificuldades no escore total e mais sintomas emocionais em relação aos demais grupos. Concluiu-se que a estratificação por dois critérios permitiu a identificação de dificuldades específicas quanto ao desempenho comportamental.
\end{abstract}

Palavras-chave: peso ao nascer, idade gestacional, comportamento, estudos de coortes

\section{Behavior of School Children Stratified by Birth Weight and Gestational Age}

\begin{abstract}
Weight and gestational age are relevant criteria for assessing the conditions of childbirth. The goal of the present study was to characterize and compare the behavior of a cohort of children stratified by two criteria: birth weight and birth weight in relation to the gestational age. At the ages of 10 and 11 years, 677 children were evaluated by the Strengths and Difficulties Questionnaire. The following statistically significant differences were observed: the low weight group had more emotional symptoms than the normal weight group; the very low weight group had more hyperactivity compared to other groups; and the small in relation to the gestational age group had more difficulties with respect to the total score and emotional symptoms in relation the other groups. It was concluded that the stratification by two criteria allowed the identification of specific difficulties in behavioral performance.
\end{abstract}

Keywords: birth weight, gestational age, behavior, cohort studies

Os avanços do conhecimento na área médica nos últimos anos contribuíram para o aumento da sobrevivência de crianças nascidas com baixo peso e pequenas para a idade gestacional (PIG) (Johnson, 2007; Milligan, 2010; Zwicker $\&$ Harris, 2008). A mortalidade dos bebês de risco diminuiu, porém a morbidade dessas crianças aumentou, o que põe em foco a necessidade de estudos que visem compreender o desenvolvimento dessas crianças mais vulneráveis (Ferraz \& Neves, 2011). No Brasil são escassos os estudos que avaliam os efeitos dos riscos biológicos e das adversidades a eles associadas ao longo do desenvolvimento dessas crianças, especialmente em estudos de coortes (Cardoso et al., 2007; Linhares, Bordin, \& Carvalho, 2004).

O desempenho comportamental tem sido considerado um importante indicador de saúde mental, usado para avaliar os desfechos do desenvolvimento infantil, especialmente para as crianças em idade escolar, devido às demandas inerentes dessa fase (Marturano \& Loureiro, 2003). O desempenho comportamental tem sido avaliado por meio de tarefas desenvolvimentais, as quais se referem a padrões socialmente

1 Apoio: FAPESP e CNPq

2 Endereço para correspondência: Rua Tenente Catão Roxo 2650, Ribeirão Preto, SP, Brasil. CEP 14051-140.E-mail: sakbc@hotmail. com esperados de desempenho dos indivíduos em diferentes áreas da sua vida e durante diferentes períodos do desenvolvimento, padrões esses que variam de acordo com a cultura, sexo, período na história e situação (Masten \& Gewirtz, 2006). Assim, é possível identificar a presença de adaptação ou de dificuldade nas trajetórias de desenvolvimento, tendo por referência comportamentos diversos, na dependência do período de desenvolvimento e das tarefas esperadas para tal período.

Alguns estudos mostram que crianças nascidas com baixo peso apresentam mais problemas comportamentais em comparação às crianças nascidas com peso normal (Aarnoudse-Moens, Weisglas-Kuperus, Goudoever, \& van Osterlaan, 2009; Bhutta, Cleves, Casey, Cradock, \& Anand, 2002; Conrad, Richman, Lindgren, \& Nopoulos, 2009; Gallo et al., 2011; Hack et al., 2004; Martins, Linhares, \& Martinez, 2005; Oliveira, Enumo, Queiroz, \& Azevedo Jr, 2011; Reijneveld et al., 2006; Rodrigues, Mello, \& Fonseca, 2006; Wiles et al., 2006). Em estudo conduzido por Gallo et al. (2011), por exemplo, foi avaliada uma coorte de 4.357 crianças, aos 11 anos, por meio do Questionário de Capacidades e Dificuldades (do inglês Strengths and Difficulties Questionnaire - SDQ), com o objetivo de verificar a associação entre o tamanho ao nascimento e 
problemas de saúde mental. Os autores observaram que as crianças nascidas com peso inferior a $2.500 \mathrm{~g}$ e de parto vaginal apresentaram maior frequência de problemas de saúde mental aos 11 anos. Scott et al. (2012) examinaram a prevalência de problemas comportamentais em crianças de 5 e 6 anos de idade provenientes de uma coorte de nascidos entre 2001 e 2003, sendo verificado que as crianças nascidas com menos de 28 semanas e com extremo baixo peso apresentaram taxa mais elevada de transtornos de atenção, de comportamentos de autorregulação e de socialização em comparação ao grupo controle, constituído por crianças nascidas com peso normal.

A literatura também aponta que as crianças nascidas PIG apresentam mais problemas comportamentais em relação às crianças nascidas adequadas para a idade gestacional (AIG). O estudo de Dahl et al. (2006), com o objetivo de avaliar os aspectos comportamentais de adolescentes nascidos com muito baixo peso por meio do Child Behavior Ckeck List (CBCL), verificou que a adequação do peso ao nascer à idade gestacional é uma variável preditora de problemas comportamentais, sendo que aqueles classificados como PIG apresentaram mais problemas comportamentais. Winchester et al. (2009) também avaliaram crianças aos 12 anos, nascidas prematuras e com diferentes morbidades perinatais quanto aos aspectos comportamentais. Os resultados obtidos por meio da Social Skills Rating Scale (SSRS) e do Teacher Report Form (TRF) mostraram que as crianças com mais morbidades perinatais (PIG, problemas neurológicos ou médicos) apresentaram resultados mais negativos em comparação às crianças nascidas a termo ou prematuras saudáveis.

Contudo, tal desfecho negativo não é exclusivo, verificando-se relatos que apontam para a ausência de dificuldades comportamentais tanto para as crianças nascidas com baixo peso (Espírito Santo, Portuguez, \& Nunes, 2009; Sabet et al., 2009) quanto para as crianças nascidas PIG (Casey, Whitside-Mansell, Barret, Bradley, \& Gargus, 2006; Hack et al., 2004). Espírito Santo et al. (2009), em estudo nacional, avaliaram, por meio da Escala Revisada de Conners para pais, 80 crianças nascidas com peso inferior a 2.500 g e nascidas com menos de 37 semanas, entre 4 e 5 anos e 11 meses, provenientes de uma coorte de nascidos em um hospital do Rio Grande do Sul. As autoras não observaram diferença significativa quanto aos aspectos comportamentais em relação ao peso ao nascer. Casey et al. (2006) avaliaram, por meio do CBCL, crianças de 8 anos, divididas em quatro grupos (crianças AIG, crianças nascidas PIG, crianças com retardo no crescimento, crianças nascidas PIG e com retardo no crescimento), com o objetivo de verificar o desempenho comportamental das mesmas. Não foram observadas diferenças significativas entre os quatro grupos analisados quanto aos aspectos comportamentais.

A diversidade de desfechos tem sido relacionada às condições ambientais que podem atenuar ou agravar as dificuldades comportamentais de crianças expostas a fatores de riscos biológicos relativos às condições de nascimento (Aylward, 2002; Ment et al., 2003; Sameroff \& Fiese, 2005). Além disso, a análise da literatura da área aponta para as dificuldades de comparação dos estudos devido às diferenças nos delineamentos adotados (Milligan, 2010; Vanderbilt \&
Gleason, 2010), na idade de avaliação das crianças (Elgen, Sommerfelt, \& Ellertsen, 2003; Ment et al., 2003; Zwicker \& Harris, 2008), nos instrumentos de avaliação utilizados (Saigal, 2000) e nos critérios de estratificação das amostras (Msall \& Park, 2008; Milligan, 2010; Sommerfelt et al., 2001).

O presente estudo se insere nessa última lacuna ao propor a caracterização e comparação do desempenho comportamental de uma mesma coorte de crianças, em idade escolar, estratificada em grupos, segundo dois critérios: 1) peso ao nascer (cinco grupos), segundo valores de referência da Organização Mundial de Saúde (OMS, 1994); e 2) peso ao nascer em relação à idade gestacional (três grupos). Desse modo, pretendeu-se contribuir com dados sobre crianças brasileiras, visando identificar as peculiaridades desses grupos quanto às dificuldades comportamentais, o que poderá instrumentar programas de prevenção e intervenção junto a essa população.

\section{Método}

\section{Participantes}

O presente estudo é parte de um projeto mais amplo, apoiado pela FAPESP (2000/09508-7), apreciado e aprovado pelo Comitê de Ética em Pesquisa do HCFMRP-USP (HCRP no 6828/2004), e realizado em duas etapas (1994 e 2004) em Ribeirão Preto-SP. Em 1994 foram coletados dados sobre todas as crianças nascidas em um período de quatro meses consecutivos, incluindo informações dos prontuários médicos relativas às características da criança (peso ao nascer, sexo e idade gestacional) e entrevistas com as mães após o nascimento para verificar as características socioeconômicas das famílias. O delineamento do estudo mais amplo e o detalhamento das características gerais da população incluída na coorte já foram previamente descritos (Cardoso et al., 2007; Silva et al., 2011). O estudo em questão é parte da segunda etapa, quando as crianças, aos 10/11 anos, foram avaliadas quanto aos aspectos comportamentais e psicológicos.

Para o estudo maior foram avaliadas 869 crianças nascidas em hospitais de Ribeirão Preto no período de maio a agosto de 1994, sendo excluídos os nascimentos gemelares e crianças que não residiam na cidade. No presente estudo, foram incluídas 677 crianças, para as quais dispunham-se de informações sobre os aspectos comportamentais e sobre as condições de nascimento. Dessas, $51 \%(n=345)$ eram do sexo masculino e $49 \%(n=332)$ do sexo feminino. Esses participantes foram agrupados segundo os dois critérios adotados.

Com base no primeiro critério, a estratificação e agrupamento dos participantes foram feitos em cinco grupos de peso ao nascer, tal como definidos pela Organização Mundial de Saúde (OMS, 1994) e caracterizado a seguir: muito baixo peso (MBP; <1500 g), incluídos 24 participantes (3,5\%); baixo peso (BP; 1500-2499 g), incluídos 124 participantes $(18,3 \%)$; peso insuficiente (PI; 2500-3000 $\mathrm{g})$, incluídos 161 participantes $(23,8 \%)$; peso normal (PN; 
3001-4250 g), incluídos 349 participantes (51,6\%); e muito alto peso (MAP; $>4250 \mathrm{~g}$ ), incluídos 19 participantes $(2,8 \%)$. Tendo por referência o segundo critério, a coorte foi estratificada e agrupada de acordo com a curva de peso ao nascer em relação à idade gestacional, proposta por Alexander, Himes, Kaufman, Mor e Kogan (1996), sendo as crianças classificadas em: pequena para a idade gestacional (PIG; abaixo do percentil 10), incluídos 140 participantes (20,7\%); adequadas para a idade gestacional (AIG; entre os percentis 10 e 90), incluídos 493 participantes $(72,8 \%)$; e grandes para a idade gestacional (GIG; acima do percentil $90)$, incluídos 44 participantes $(6,5 \%)$.

\section{Instrumentos}

Foram utilizados os seguintes instrumentos:

a) Questionário de Capacidades e Dificuldades SDQ (Fleitlich, Córtazar, \& Goodman, 2000; Goodman, 1997; Goodman, 2001): avalia a presença ou ausência de indicadores comportamentais, visando o rastreamento de problemas de saúde mental infantil, de acordo com os critérios estabelecidos pelo DSM-IV. Esse questionário é um instrumento de uso livre (www.sdqinfo.com), de rápida aplicação e composto por cinco escalas: sintomas emocionais, problemas de conduta, hiperatividade, problemas de relacionamento com colegas e comportamento pró-social. Adotou-se a versão respondida pelos pais.

$\mathrm{Na}$ aplicação do questionário, os pais devem assinalar, para cada sentença: falso, mais ou menos verdadeiro ou verdadeiro. As respostas são codificadas em 0,1 e 2 pontos e em cada escala os pontos são somados, sendo que para cada uma das cinco escalas a pontuação pode variar de 0 a 10 pontos. A pontuação total do instrumento para a obtenção do escore total de dificuldades inclui as escalas de sintomas emocionais, problemas de conduta, problemas de relacionamento com colegas e hiperatividade, totalizando, no máximo, 40 pontos, visto que a escala de comportamento pró-social não é incluída nesse escore, caracterizando-se como um indicador de capacidade. Destaca-se que quanto maior a pontuação obtida nas escalas e no escore total, maior a indicação de problemas comportamentais, exceto para a escala de comportamento pró-social, onde quanto maior a pontuação, menor a dificuldade.

A tradução e adaptação às características socioculturais brasileiras do SDQ e os estudos apresentando bons índices de validade (validade discriminativa $\chi 2=13.1 ; \mathrm{p}<0.001$ ) e fidedignidade (teste de amostras pareadas, $\mathrm{p}=0,148$ ) do instrumento no Brasil foram descritos por Woerner et al. (2004).

b) Questionário Complementar: questões selecionadas do estudo maior, obtidas por meio de consulta aos prontuários médicos e de entrevistas com as mães logo após o nascimento de seus filhos. Para os objetivos deste estudo, foram selecionadas informações relativas às crianças (sexo, peso ao nascer, idade gestacional, condições de saúde) e às famílias das crianças (situação conjugal da mãe, escolaridade da mãe e do pai, ocupação do chefe da família, número de moradores residindo no mesmo domicílio que a criança e classificação socioeconômica).

\section{Procedimento}

A localização das crianças na coleta dos anos de 2004/2005 ocorreu por meio de uma consulta ao cadastro geral de alunos gerenciado pela Secretaria Estadual da Educação. A partir da localização das crianças nas escolas, foi feito contato telefônico com as famílias para explicar os objetivos do estudo e solicitar a autorização para a realização das diferentes etapas do estudo maior. As avaliações foram realizadas por uma equipe treinada de psicólogos e de técnicos em pesquisa.

Após a assinatura do Termo de Consentimento Livre e Esclarecido, os pais ou responsáveis, em sessão individual, responderam por escrito ao SDQ, na presença de uma psicóloga. Os dados relativos às condições clínicas das crianças e socioeconômicas das famílias tiveram por fonte os prontuários médicos e informações das mães, que responderam ao Questionário Complementar, aplicado por um auxiliar de pesquisa do projeto maior no momento do nascimento das crianças.

\section{Análise de dados}

Os dados obtidos por meio do SDQ foram codificados de acordo com as proposições de Goodman (1997). Para cada uma das cinco escalas e para o escore total, o instrumento fornece notas relativas às categorias normal, limítrofe e anormal. Para os objetivos deste estudo, optou-se por agrupar as classificações normal e limítrofe (escore menor que 16) em contraposição à classificação anormal (escore maior que 16), e adotou-se para as classificações do escore total e das escalas a nomenclatura "Sem Dificuldade" (SD), para as categorias normal e limítrofe, e "Com Dificuldade" (CD), para referir-se à classificação anormal, relativa à faixa clínica.

As informações relativas às características biológicas e clínicas das crianças (sexo, tipo de parto, tempo de internação, amamentação) e socioeconômicas das famílias (escolaridade da mãe e do pai, ocupação do chefe de família, estado civil da mãe, número de pessoas vivendo no mesmo domicílio e classificação socioeconômica das famílias) foram quantificadas sob a forma de frequência e tabuladas em planilhas. Em relação à classificação socioeconômica das famílias das crianças, foi utilizado o Critério de Classificação Econômica Brasil (CCEB), com correção e interpretação proposta pela Associação Brasileira de Empresas de Pesquisa (ABEP, 2008), sendo os níveis A1, A2, B1, B2 os mais favorecidos e os outros níveis classificados como menos favorecidos.

Para o tratamento dos dados adotou-se estatística nãoparamétrica devido à distribuição não normal das variáveis (Teste de Shapiro-Wilks, $\mathrm{p}>0,10$ ) e adotou-se, em todas as comparações, o nível de significância de $p \leq 0,05$.

Procedeu-se à análise descritiva calculando-se as frequências absoluta e relativa para as variáveis discretas. Para as comparações entre os grupos em relação às variáveis biológicas, características clínicas das crianças e socioeconômicas das famílias, foram utilizados os Testes do Qui-Quadrado ou Teste Exato de Fisher. 
Para as comparações relativas ao desempenho comportamental, avaliadas pelo SDQ, entre os cinco grupos de peso ao nascer ( $1^{\circ}$ critério) e os três grupos de peso ao nascer em relação à idade gestacional $\left(2^{\circ}\right.$ critério $)$, adotou-se o Teste de Kruskal-Wallis, com post-hoc de Dunn.

\section{Resultados}

Considerando o objetivo do presente estudo, os resultados serão apresentados para cada critério adotado na estratificação da amostra.

\section{Características biológicas e clínicas das crianças e socioeconômicas das famílias $-\mathbf{1}^{\circ}$ critério}

Os dados de caracterização serão apresentados exclusivamente de modo descritivo. Verificou-se, quando da estratificação da amostra em cinco grupos de peso ao nascer, uma distribuição homogênea em relação ao sexo para três grupos de peso ao nascer (BP, PI, PN) e o predomínio do sexo masculino para dois grupos (MBP-62,5\% e MAP$78,9 \%$ ). Foram verificados mais meninos para o grupo MAP, em comparação aos grupos BP, PI e PN, sendo essa diferença estatisticamente significativa.

No que diz respeito à idade gestacional, a maior parte das crianças nasceu a termo $(77,3 \%)$. O grupo MBP teve significativamente mais nascimentos pré-termo $(<37$ semanas) em relação aos grupos BP, PI, PN e MAP. Quanto ao peso ao nascer em relação à idade gestacional, observou-se que nos grupos PN e MAP não foram incluídas crianças classificadas como PIG. Nos dois grupos de baixo peso, a maioria das crianças foi classificada como PIG (MBP - 54,2\% e BP - 67,7\%), sendo que o grupo MBP teve significativamente mais crianças PIG do que AIG em relação ao grupo PI.

Sobre as características clínicas das crianças, predominou o tempo mais curto de internação após o nascimento para quatro grupos de peso (BP, PI, PN e MAP), sendo que as crianças do grupo MBP ficaram significativamente mais dias internadas em relação às crianças dos demais grupos.

Não foi observada diferença significativa entre os grupos em relação à presença de complicações respiratórias na infância. Em relação à amamentação, observou-se que $94,4 \%$ do total de crianças da coorte tiveram acesso ao leite materno e que as crianças do grupo PN tiveram significativamente mais acesso ao leite materno em comparação aos grupos MBP e BP.

Quanto às características socioeconômicas das famílias das crianças, em todos os grupos de peso predominou as mães casadas ou amasiadas ( $85,2 \%$ das mães), sem diferença significativa entre os grupos. Com relação à escolaridade das mães e dos pais, não foram observadas diferenças significativas entre os grupos e observou-se que mais de $60 \%$ dessas mulheres e pelo menos $50 \%$ dos pais estudaram de zero a oito anos.

Não foram observadas diferenças significativas entre os grupos de peso quanto à ocupação do chefe de família, predominando a categoria manual qualificada ou semiqualificada para todos os grupos. Quanto ao nível socioeconômico, mais de $55 \%$ das famílias da coorte de crianças foram incluídas nas classes $\mathrm{C} 1, \mathrm{C} 2$, D e E (MBP 66,7\%; BP - 64,2\%; PI - 59,7\%; PN - 59,7\%; MAP - 57,9\%). No grupo MAP, $21,1 \%$ das famílias foram incluídas nas classes A1 e A2, enquanto menos de $7 \%$ das famílias dos demais grupos foram incluídas nessa categoria; contudo, não foram observadas diferenças significativas entre os cinco grupos de peso.

Quanto ao número de pessoas que moram na casa com a criança, mais de $60 \%$ das famílias da coorte referiu morar com até quatro pessoas em suas casas, e apenas no grupo MAP significativamente mais famílias referiram morar com cinco ou mais pessoas em comparação ao grupo PN.

\section{Perfil comportamental das crianças $-1^{\circ}$ critério}

A Tabela 1 apresenta o desempenho comportamental das crianças da coorte estudada, estratificadas em cinco grupos de peso ao nascer.

Tabela 1. Desempenho comportamental: distribuição das crianças incluídas na faixa clínica ("Com Dificuldade" no SDQ) de acordo com o peso ao nascer

\begin{tabular}{lccccc}
\hline & \multicolumn{5}{c}{ GRUPOS } \\
\cline { 2 - 5 } \multicolumn{1}{c}{ SDQ } & MBPF $(\%)$ & BPf $(\%)$ & PI f $(\%)$ & PN f $(\%)$ & MAP f $(\%)$ \\
\hline Escore Total & $12(50,0)$ & $54(43,5)$ & $58(36,0)$ & $129(37,0)$ & $4(21,1)$ \\
Sintomas Emocionais & $9(37,5)$ & $78(62,9)$ & $95(59,0)$ & $173(49,6)$ & $8(42,1)$ \\
Problemas de Conduta & $5(20,8)$ & $44(35,5)$ & $43(26,7)$ & $127(36,4)$ & $5(26,3)$ \\
Hiperatividade & $12(50,0)$ & $35(28,2)$ & $39(24,2)$ & $82(23,5)$ & $1(5,3)$ \\
Problema com Colegas & $7(29,2)$ & $37(29,8)$ & $45(28,0)$ & $88(25,2)$ & $4(21,1)$ \\
Comportamento Pró-Social & $0(0)$ & $6(4,8)$ & $8(5,0)$ & $11(3,2)$ & $0(0)$ \\
\hline
\end{tabular}

Nota. MBP-muito baixo peso, BP-baixo peso, PI-peso insuficiente, PN-peso normal, MAP-muito alto peso.

Teste do Qui-quadrado ou Teste Exato de Fisher $(\mathrm{p} \leq 0,05)$. 
Verificou-se que os grupos MBP e BP foram os que tiveram maior frequência de crianças com problemas comportamentais, segundo relato dos pais. Notou-se uma diminuição na frequência de crianças com dificuldade comportamental na medida em que o peso ao nascer foi maior, mas não foram observadas diferenças significativas no escore total de dificuldades em relação aos cinco grupos de peso.

$\mathrm{Na}$ escala de sintomas emocionais, o grupo BP teve significativamente mais crianças com dificuldades em comparação às crianças dos grupos $\mathrm{MBP}$ e $\mathrm{PN}$. O grupo PI também teve significativamente mais crianças com sintomas emocionais em comparação ao grupo MBP.

Quanto à escala de hiperatividade, observou-se que as crianças do grupo MBP apresentaram significativamente mais dificuldades em comparação às crianças dos grupos BP, PI, PN e MAP.

Com relação às escalas de problemas de conduta, problemas de relacionamento com colegas e de comportamento pró-social, predominou a ausência de problemas de comportamento com distribuição semelhante e sem diferenças significativas entre os grupos de peso.

\section{Análises de comparação do desempenho comportamental $-1^{\circ}$ critério}

A Tabela 2 apresenta os resultados das análises de comparação do desempenho comportamental para os cinco grupos de peso ao nascer.

Observaram-se diferenças estatisticamente significativas para as escalas de sintomas emocionais e de hiperatividade. $\mathrm{Na}$ escala de sintomas emocionais, o grupo BP teve significativamente mais crianças com dificuldades em relação às crianças dos grupos MBP e PN. Quanto à escala de hiperatividade, as crianças do grupo MBP apresentaram significativamente mais dificuldades em relação às crianças dos grupos BP, PI, PN e MAP.

Não foram observadas diferenças significativas quanto ao desempenho comportamental para o escore total e para as escalas de problemas de conduta, problemas de relacionamento com colegas e comportamento pró-social entre os cinco grupos de peso.

No próximo tópico são apresentados os dados relacionados ao segundo critério, peso ao nascer em relação à idade gestacional.

\section{Características biológicas e clínicas das crianças e socioeconômicas das famílias $-2^{\circ}$ critério}

Os dados de caracterização serão apresentados apenas de modo descritivo. Verificou-se distribuição homogênea em relação ao sexo das crianças para os grupos AIG e GIG (54,4\% e 59,1\%, respectivamente), enquanto, o grupo PIG teve significativamente menos meninos em comparação ao grupo AIG e ao grupo GIG.

Quanto à idade gestacional, mais de $70 \%$ das crianças nasceram a termo nos três grupos e o nascimento pré-termo foi significativamente maior no grupo PIG em comparação ao grupo GIG.

Notou-se, para os três grupos, o predomínio de um tempo mais curto de internação no hospital após o nascimento (86,8\%), mas as crianças do grupo PIG ficaram significativamente mais dias internadas em comparação às crianças dos grupos AIG e GIG.

Sobre a presença de complicações respiratórias na infância, observou-se uma frequência próxima nos grupos

Tabela 2. Desempenho comportamental das crianças: comparações dos cinco grupos de peso ao nascer.

\begin{tabular}{|c|c|c|c|c|c|c|c|}
\hline \multirow[b]{2}{*}{ SDQ } & \multicolumn{5}{|c|}{ GRUPOS } & \multirow[b]{2}{*}{$\mathrm{p}$} & \multirow[b]{2}{*}{$\begin{array}{c}\text { Direção das } \\
\text { diferenças } \\
\text { entre grupos }\end{array}$} \\
\hline & $\begin{array}{l}\text { MBP } \\
\text { Média } \\
\text { (DP) }\end{array}$ & $\begin{array}{c}\text { BP } \\
\text { Média } \\
\text { (DP) }\end{array}$ & $\begin{array}{c}\text { PI } \\
\text { Média } \\
\text { (DP) }\end{array}$ & $\begin{array}{c}\text { PN } \\
\text { Média } \\
\text { (DP) }\end{array}$ & $\begin{array}{l}\text { MAP } \\
\text { Média } \\
\text { (DP) }\end{array}$ & & \\
\hline Escore Total & $\begin{array}{l}16,21 \\
(6,09)\end{array}$ & $\begin{array}{l}15,86 \\
(7,44)\end{array}$ & $\begin{array}{l}14,52 \\
(7,19)\end{array}$ & $\begin{array}{l}14,22 \\
(6,88)\end{array}$ & $\begin{array}{l}11,79 \\
(7,46)\end{array}$ & 0,20 & \\
\hline Sintomas Emocionais & $\begin{array}{c}4,83 \\
(2,82)\end{array}$ & $\begin{array}{c}5,37 \\
(2,65)\end{array}$ & $\begin{array}{c}5,24 \\
(2,78)\end{array}$ & $\begin{array}{c}4,82 \\
(2,40)\end{array}$ & $\begin{array}{c}4,00 \\
(2,58)\end{array}$ & $0,02 *$ & $\begin{array}{l}\mathrm{BP}>\mathrm{MBP} \text { e } \\
\mathrm{PN} \mathrm{PI}>\mathrm{PN}\end{array}$ \\
\hline Problemas de Conduta & $\begin{array}{c}2,50 \\
(2,16)\end{array}$ & $\begin{array}{c}2,94 \\
(2,37)\end{array}$ & $\begin{array}{l}2,45 \\
(2,20)\end{array}$ & $\begin{array}{c}2,79 \\
(2,24)\end{array}$ & $\begin{array}{c}2,47 \\
(2,56)\end{array}$ & 0,13 & \\
\hline Hiperatividade & $\begin{array}{c}6,08 \\
(2,30)\end{array}$ & $\begin{array}{c}4,89 \\
(3,03)\end{array}$ & $\begin{array}{c}4,35 \\
(2,70)\end{array}$ & $\begin{array}{c}4,41 \\
(2,80)\end{array}$ & $\begin{array}{c}3,63 \\
(2,43)\end{array}$ & $0,01 * *$ & $\begin{array}{c}\mathrm{MBP}>\mathrm{BP}, \\
\mathrm{PI}, \mathrm{PN} \text { e MAP }\end{array}$ \\
\hline Problema com Colegas & $\begin{array}{c}2,83 \\
(1,81)\end{array}$ & $\begin{array}{c}2,66 \\
(2,02)\end{array}$ & $\begin{array}{c}2,55 \\
(1,99)\end{array}$ & $\begin{array}{c}2,25 \\
(1,99)\end{array}$ & $\begin{array}{c}1,68 \\
(2,35)\end{array}$ & 0,83 & \\
\hline Comportamento Pró-Social & $\begin{array}{c}8,38 \\
(1,05)\end{array}$ & $\begin{array}{c}8,27 \\
(1,80)\end{array}$ & $\begin{array}{c}8,32 \\
(1,85)\end{array}$ & $\begin{array}{c}8,48 \\
(1,71)\end{array}$ & $\begin{array}{c}9,05 \\
(1,12)\end{array}$ & 0,54 & \\
\hline
\end{tabular}

Nota. MBP-muito baixo peso, BP-baixo peso, PI-peso insuficiente, PN-peso normal, MAP-muito alto peso. Teste do Quiquadrado ou Teste Exato de Fisher $(\mathrm{p} \leq 0,05)$. 
AIG e GIG e maior frequência no grupo PIG, sem diferenças significativas entre os grupos.

Não foi observada diferença significativa entre os grupos para a variável amamentação. Nos três grupos predominaram as mães casadas ou amasiadas (85,2\% das mães), sem diferença significativa entre os grupos.

Quanto à escolaridade das mães, observou-se que mais da metade estudou de zero a oito anos para os grupos PIG $(70,5 \%)$ e AIG $(60,6 \%)$. As mães do grupo AIG e do grupo GIG apresentaram significativamente mais anos de estudo em relação às mães do grupo PIG. Em relação à escolaridade dos pais ou chefes de família, mais de $40 \%$ dos pais estudaram de zero a oito anos (PIG - 70,2\%; AIG - 58,5\% e GIG - 48,6\%). Verificou-se que os pais ou chefes de família do grupo PIG estudaram significativamente menos que os pais dos grupos AIG e GIG.

Para a ocupação do chefe de família, notou-se o predomínio da categoria manual qualificada ou semiqualificada para todos os grupos, com frequência variando de $61,7 \%$ a $64,4 \%$ para essa categoria, sem diferença significativa entre os grupos.

Em relação à classe econômica, não foram observadas diferenças significativas nas comparações entre os grupos. Observou-se que mais de $50 \%$ das famílias foram incluídas nas classes menos favorecidas, C1, C2, D e E (PIG-70,5\%; AIG-59,3\% e GIG-50\%), sendo o grupo PIG o de maior frequência nessas classificações. Nas classes A1 e A2 foram incluídas mais famílias do grupo GIG $(11,4 \%)$ em comparação aos outros dois grupos (PIG - 3,6\% e AIG - 7\%).

Tabela 3. Desempenho comportamental: distribuição das crianças incluídas na faixa clínica (“Com Dificuldade" no SDQ) de acordo com o peso ao nascer em relação à idade gestacional.

\begin{tabular}{lccc}
\hline \multirow{2}{*}{ SDQ } & \multicolumn{3}{c}{ GRUPOS } \\
\cline { 2 - 4 } & PIG & AIG & GIG \\
& $\mathrm{f}(\%)$ & $179(36,3)$ & $13(29,5)$ \\
\hline Escore Total & $65(46,4)$ & $249(50,5)$ & $18(40,9)$ \\
Sintomas Emocionais & $96(68,6)$ & $158(32,0)$ & $17(38,6)$ \\
Problemas de Conduta & $49(35,0)$ & $122(24,7)$ & $10(22,7)$ \\
Hiperatividade & $37(26,4)$ & $125(25,4)$ & $12(27,3)$ \\
Problema com Colegas & $44(31,4)$ & $21(4,3)$ & $0(0)$ \\
Comportamento Pró-Social & $4(2,9)$ & & \\
\hline
\end{tabular}

Nota. PIG-pequeno para a idade gestacional, AIG-adequado para a idade gestacional, GIG-grande para a idade gestacional. Teste do Qui-quadrado ou Teste Exato de Fisher $(\mathrm{p} \leq 0,05)$.

Tabela 4. Desempenho comportamental das crianças: comparações dos três grupos de peso ao nascer em relação à idade gestacional

\begin{tabular}{|c|c|c|c|c|c|}
\hline \multirow[b]{2}{*}{ SDQ } & \multicolumn{5}{|c|}{ GRUPOS } \\
\hline & $\begin{array}{l}\text { PIG } \\
\text { Média } \\
\text { (DP) }\end{array}$ & $\begin{array}{l}\text { AIG } \\
\text { Média } \\
\text { (DP) }\end{array}$ & $\begin{array}{l}\text { GIG } \\
\text { Média } \\
(\mathrm{DP})\end{array}$ & $\mathrm{p}$ & $\begin{array}{c}\text { Direção das } \\
\text { diferenças } \\
\text { entre grupos }\end{array}$ \\
\hline Escore Total & $\begin{array}{l}16,35 \\
(7,00)\end{array}$ & $\begin{array}{l}14,22 \\
(6,96)\end{array}$ & $\begin{array}{l}13,18 \\
(7,78)\end{array}$ & $0,04 *$ & $\begin{array}{c}\mathrm{PIG}>\mathrm{AIG} \\
\mathrm{GIG}\end{array}$ \\
\hline Sintomas Emocionais & $\begin{array}{c}5,63 \\
(2,53)\end{array}$ & $\begin{array}{c}4,91 \\
(2,57)\end{array}$ & $\begin{array}{c}3,98 \\
(2,22)\end{array}$ & $0,00 * * *$ & $\begin{array}{c}\mathrm{PIG}>\mathrm{AIG} \\
\mathrm{GIG}\end{array}$ \\
\hline Problemas de Conduta & $\begin{array}{c}3,06 \\
(2,33)\end{array}$ & $\begin{array}{c}2,61 \\
(2,22)\end{array}$ & $\begin{array}{c}2,84 \\
(2,52)\end{array}$ & 0,59 & \\
\hline Hiperatividade & $\begin{array}{c}4,91 \\
(2,78)\end{array}$ & $\begin{array}{c}4,43 \\
(2,80)\end{array}$ & $\begin{array}{c}4,30 \\
(2,98)\end{array}$ & 0,85 & \\
\hline Problema com Colegas & $\begin{array}{c}2,80 \\
(2,04)\end{array}$ & $\begin{array}{c}2,32 \\
(1,95)\end{array}$ & $\begin{array}{c}2,07 \\
(2,37)\end{array}$ & 0,35 & \\
\hline Comportamento Pró-Social & $\begin{array}{c}8,41 \\
(1,66)\end{array}$ & $\begin{array}{c}8,40 \\
(1,78)\end{array}$ & $\begin{array}{c}8,57 \\
(1,40)\end{array}$ & 0,29 & \\
\hline
\end{tabular}

Nota. PIG-pequeno para a idade gestacional, AIG-adequado para a idade gestacional, GIG-grande para a idade gestacional. Teste de Kruskal-Wallis $(\mathrm{p} \leq 0,05)$. 
Quanto ao número de pessoas que moram na casa com a criança, a maioria das famílias da coorte referiu morar com até quatro pessoas em suas casas, sem diferença significativa entre os grupos.

\section{Perfil comportamental das crianças - $2^{\circ}$ critério}

A Tabela 3 apresenta o desempenho comportamental das crianças da coorte estudada, estratificada em três grupos de peso ao nascer em relação à idade gestacional.

Observou-se que o grupo PIG incluiu significativamente mais crianças classificadas com dificuldade no escore total em comparação ao grupo AIG e GIG. Quanto à escala de sintomas emocionais, as crianças do grupo PIG apresentaram significativamente mais dificuldades em comparação às crianças dos grupos AIG e GIG.

$\mathrm{Na}$ escala de problemas de conduta, de hiperatividade, de problemas de relacionamento com colegas e de comportamento pró-social, não foram verificadas diferenças significativa entre os grupos.

Análises de comparação do desempenho comportamental $-2^{\circ}$ critério

A Tabela 4 apresenta as análises de comparação do desempenho comportamental para os três grupos de peso ao nascer em relação à idade gestacional.

Observou-se que as crianças do grupo PIG apresentaram significativamente mais dificuldades comportamentais no escore total em comparação às crianças dos grupos AIG e GIG. As crianças do grupo PIG também mostraram mais dificuldades na escala de sintomas emocionais em relação às crianças dos grupos AIG e GIG.

Nas comparações entre os grupos quanto às escalas de problemas de conduta, hiperatividade, problemas de relacionamento com colegas e comportamento pró-social, não foram observadas diferenças estatisticamente significativas.

\section{Discussão}

Verificou-se que as crianças nascidas com baixo peso apresentaram mais problemas comportamentais em comparação às crianças nascidas com peso normal, da mesma forma que as crianças PIG apresentaram mais problemas comportamentais em relação às nascidas AIG. Pelos dois critérios de estratificação da coorte, os dados obtidos são concordantes com os dados da literatura que apontam para a vulnerabilidade desses grupos (Aarnoudse-Moens et al., 2009; Bhutta et al., 2002; Dahl et al., 2006; Martins et al., 2005; Msall \& Park, 2008; Oliveira et al., 2011). Constatou-se ainda, que as crianças nascidas com baixo peso apresentaram mais dificuldades na escala de sintomas emocionais em comparação aos nascidos com peso normal, mas não apresentaram mais problemas comportamentais avaliados pelo escore total, enquanto as crianças nascidas PIG apresentaram mais sintomas emocionais e também mais dificuldades quanto ao escore total em comparação às nascidas AIG.

A presença de mais sintomas emocionais para as crianças nascidas com baixo peso é concordante com o relato de estudos prévios que descrevem tais crianças com mais problemas internalizantes em comparação às crianças nascidas com peso normal (Aarnoudse-Moens et al., 2009; Hayes \& Sharif, 2009; Martins et al., 2005; Reijneveld et al., 2006;). Uma peculiaridade foi que o grupo BP apresentou mais dificuldade nessa escala em relação ao grupo MBP, apontado em outros estudos como o grupo com mais dificuldades (Bhutta et al., 2002; Reijneveld et al., 2006).

Considerando que as competências comportamentais das crianças são influenciadas pela interação de diversos fatores relacionados à saúde da criança, ao ambiente familiar, às condições sociais e ao suporte da comunidade (Msall \& Park, 2008; Vanderbilt \& Gleason, 2010), destacam-se dois fatores que podem ter favorecido maior risco às crianças do grupo BP nessa coorte. Esse grupo teve significativamente mais meninas em comparação aos outros grupos de peso, e estas são, reconhecidamente, mais propensas a apresentar problemas internalizantes em comparação aos meninos (Msall \& Park, 2008; Reijneveld et al., 2006). Além disso, apesar da diferença não ter sido estatisticamente significativa, a escolaridade das mães dessas crianças foi menos privilegiada, predominando a baixa escolaridade $(66,1 \%)$, enquanto que para as mães do grupo MBP, predominou mais anos de estudo $(54,2 \%)$. Observou-se que o grupo PI também apresentou significativamente mais dificuldades na escala de sintomas emocionais em comparação ao grupo $\mathrm{PN}$, possivelmente porque nesse grupo também tinha significativamente mais meninas e mais crianças nascidas prematuras e PIG em comparação ao grupo PN.

Em relação às crianças nascidas $\mathrm{PIG}$, alguns estudos apontaram que essas crianças não apresentam mais problemas internalizantes (Dahl et al., 2006; Hack et al., 2004), diferentemente do observado no presente estudo, o que evidencia a importância de explicitar os aspectos combinados quando da comparação de resultados. No estudo de Dahl et al. (2006) predominou o grupo de crianças nascidas com muito baixo peso, enquanto no presente estudo apenas 3\% das incluídas eram nascidas nessa faixa de peso, predominando o baixo peso. E, no estudo de Hack et al. (2004), o resultado mais positivo pode estar relacionado com a idade de avaliação, já que os problemas comportamentais tendem a diminuir com a idade (Wiles et al., 2006).

Quanto à escala de hiperatividade, as crianças do grupo MBP apresentaram significativamente mais dificuldades em comparação aos outros grupos de peso, o que é concordante com estudos prévios que relatam que as crianças nascidas com muito baixo peso apresentam mais dificuldades nessa área em comparação às crianças nascidas com peso normal (Conrad et al., 2009; Hack et al., 2004; Msall \& Park, 2008). Por outro lado, no grupo PIG não foi observada mais dificuldade nessa escala, o que coloca em destaque uma peculiaridade desse grupo, e a relevância da comparação de critérios de estratificação com a mesma amostra.

Diversos estudos sugerem que a hiperatividade é mais frequente em crianças nascidas prematuras (Bhutta et al., 2002) e com muito baixo peso (Msall \& Park, 2008; Rodrigues et al., 2006). Assim, a presença de mais dificuldades nessa escala apenas para o grupo MBP pode estar relacionada à homogeneidade desse grupo quanto aos fatores de risco do muito baixo peso ao nascer e da prematuridade 
$(91,7 \%)$ e à heterogeneidade do grupo PIG para esses fatores (9,3\% nascidos MBP e 29,3\% prematuros). Entretanto, no estudo de Oliveira et al. (2011), as crianças com baixo peso e prematuras não apresentaram problemas relacionados à hiperatividade quando comparadas ao grupo de crianças de peso normal e nascidas a termo, avaliadas pelo CBCL, na idade pré-escolar. Ressalta-se, contudo, a dificuldade de comparação entre estudos com diferentes delineamentos, instrumentos e idades de avaliação.

Com base no segundo critério, identificou-se o grupo PIG como o mais vulnerável às dificuldades comportamentais e emocionais. Vale ressaltar que além do próprio risco de ser PIG, nessa coorte essas crianças também estavam expostas a condições mais adversas em comparação aos outros grupos. O grupo PIG apresentou significativamente mais crianças nascidas prematuras em comparação ao grupo GIG, e $9,3 \%$ e $60 \%$ nasceram com muito baixo peso e baixo peso, respectivamente. Também ficaram significativamente mais dias internadas e a escolaridade dos pais foi significativamente menor em comparação aos outros grupos, fatores esses que podem ter contribuído para o resultado mais negativo desse grupo.

Quanto às dificuldades comportamentais avaliadas pelas escalas de problemas de conduta e de problemas de relacionamento com colegas foi verificada, com os dois critérios de estratificação, a ausência de diferenças significativas entre os grupos. Assim, pode-se dizer que não foi verificada a presença de mais problemas externalizantes para as crianças nascidas com baixo peso e PIG em comparação às crianças nascidas com peso normal e AIG, como relatado por alguns estudos (Bhutta et al., 2002; Dahl et al., 2006; Msall \& Park, 2008). Também não foram observadas diferenças significativas entre os grupos para a escala de comportamento pró-social, indicadora de recursos de socialização, sendo a presença de dificuldades nessa escala inferior a $5 \%$ para todos os grupos.

Os resultados decorrentes da análise que estratificou a amostra de acordo com o peso ao nascer em relação à idade gestacional apontaram para a presença de mais dificuldades comportamentais e emocionais para as crianças nascidas PIG. Tais dados sugerem que as crianças nascidas PIG apresentaram aos 10/11 anos mais riscos decorrentes dessa condição de nascimento, caracterizando um impacto mais negativo dessa condição para o desenvolvimento dessas crianças. Portanto, esse grupo de crianças requer mais atenção, quando da proposição de práticas preventivas e de programas de acompanhamento do desenvolvimento de crianças expostas a adversidades quando do nascimento.

O presente estudo, ao caracterizar e comparar o desempenho comportamental das crianças, na idade escolar, utilizando a mesma amostra e dois diferentes critérios de estratificação da coorte quando da condição de nascimento, permitiu a comparação das peculiaridades dos mesmos.

Quando da utilização do critério que considerou o peso ao nascer, foram verificadas diferenças significativas apenas para as escalas de sintomas emocionais e hiperatividade, possivelmente devido aos fatores de proteção observados na coorte (condições favoráveis de saúde das crianças e da escolaridade da mãe) e, em especial, porque apenas 3,5\% das crianças apresentavam muito baixo peso, faixa de peso que tende a apresentar maiores déficits comportamentais. Nesse critério, as crianças foram distribuídas em grupos mais homogêneos quanto ao peso ao nascer, tornando possível obter resultados mais específicos e identificando-se os grupos em que as dificuldades se concentraram: para o grupo MBP, a hiperatividade, e para os grupos BP e PI, os sintomas emocionais.

Comparando-se os achados para os dois critérios, verificou-se que ambos detectaram mais sintomas emocionais para as crianças dos grupos BP e PIG, enquanto que a presença de mais hiperatividade foi verificada apenas para $\mathrm{O}$ grupo MBP ( $1^{\circ}$ critério $)$ e a identificação de mais dificuldades no escore total foi observada somente para as crianças do grupo PIG ( $2^{\circ}$ critério). No caso da hiperatividade, foi possível observar o efeito das variáveis peso ao nascer e prematuridade, devido à maior homogeneidade do grupo de MBP nesses aspectos, em comparação ao grupo PIG. Com relação ao escore total, notou-se a influência de múltiplos fatores para o desempenho comportamental, visto que o grupo PIG ficou mais exposto a fatores de risco, tais como: prematuridade $(29,3 \%)$, muito baixo e baixo peso $(69,3 \%)$, baixo nível socioeconômico das famílias (70\%) e pouca escolaridade (apenas quatro anos - 50,7\%).

Quanto à escala de sintomas emocionais, nos dois critérios de estratificação da amostra, foram verificadas mais dificuldade para as crianças dos grupos BP e PIG, sugerindo um efeito tanto do baixo peso ao nascer quanto da condição PIG nesse aspecto do desempenho comportamental. A avaliação comportamental por meio dos pais pode ter contribuído para esse resultado, visto que os pais podem ter uma visão dos filhos como mais frágeis devido às condições adversas enfrentadas no nascimento. Segundo Vanderbilt e Gleason (2010), faz-se necessário ter cautela ao se utilizar as avaliações realizadas por pais, pois tais avaliações refletem a percepção dos adultos sobre os padrões comportamentais. Em estudos futuros seria importante considerar também as avaliações comportamentais feitas pelas próprias crianças; nesse sentido, o SDQ é instrumento particularmente útil por ter também uma versão para crianças e jovens.

Em estudos futuros sugere-se que as crianças nascidas PIG sejam estratificadas também de acordo com o peso ao nascer, a fim de verificar como se mostra o desempenho comportamental das crianças PIG de MBP, PIG de BP e PIG de PI, além de verificar como variáveis clínicas e socioeconômicas concorrem para tal perfil.

Consideraram-se algumas limitações do estudo, a saber: a inclusão de um pequeno número de crianças nascidas com muito baixo peso $(<1.500 \mathrm{~g})$ e com muito alto peso $(>4.250$ $\mathrm{g})$; o acesso às famílias apenas por meio da escola, o que possivelmente contribuiu para uma amostra mais saudável; a avaliação comportamental coletada apenas com um informante; e a ausência de informação sistemática sobre a presença de indicadores de risco neonatal, o que limitou a análise relativa às condições de saúde.

A principal contribuição deste estudo refere-se à utilização, com a mesma amostra, de dois diferentes critérios de estratificação, para a realização das comparações relativas ao desempenho comportamental, visto que a literatura tem abordado a dificuldade de comparação dos dados existentes devido às diferenças metodológicas, aos critérios de 
estratificação da amostra e à variabilidade de instrumentos utilizados (Aylward, 2002; Milligan, 2010). Assim, neste estudo, as mesmas crianças, de uma única coorte, foram avaliadas pelo mesmo instrumento e comparadas por meio de dois diferentes critérios, viabilizando as comparações entre os critérios adotados.

O grande número de crianças avaliadas em um curto período de tempo também fortalece os resultados apresentados, pois favoreceu que possíveis fatores ambientais fossem melhor controlados, maximizando as vantagens do delineamento de coorte. Outra contribuição foi a avaliação do comportamento por meio de instrumento aferido para o Brasil, o que favorece a fidedignidade e validade da medida.

Concluiu-se que a estratificação em cinco grupos de peso ao nascer favoreceu a visualização dos efeitos diferenciados da variável peso ao nascer e a estratificação do peso ao nascer em relação à idade gestacional caracterizou a condição PIG como a de maior impacto para o desenvolvimento dessas crianças. Tais resultados contribuem para a prática, podendo nortear os programas de acompanhamento do desenvolvimento infantil e para a pesquisa, sugerindo a necessidade de estudos que tenham como foco as condições de desenvolvimento de tais crianças.

\section{Referências}

Aarnoudse-Moens, C. S. H., Weisglas-Kuperus, N., Goudoever, J. B. V., \& van Osterlaan, J. (2009). Meta-analysis of neurobehavioral outcomes in very preterm and/or very low birth weigth children. Pediatrics, 124(2), 717-728.

Alexander, G. R., Himes, J. H., Kaufman, R. B., Mor, J., \& Kogan, M. (1996). A United States national reference for fetal growth. Obstetric Gynecoly, 87(2), 163-168.

Associação Brasileira de Empresas de Pesquisa - ABEP (2008). Questionário de Classificação Socioeconômico: 2008. Retrieved from http://www.abep.org/novo/CMS/Utils/ FileGenerate. $\operatorname{ash} x$ ? id $=22$

Aylward, G. P. (2002). Methodological issues in outcome studies of at-risk infants. Journal of Pediatric Psychology, 27(1), 37-45.

Bhutta, A. T., Cleves, M. A., Casey, P. H., Cradock, M. M., \& Anand, K. J. (2002). Cognitive and behavioral outcomes of school-aged children who were born preterm: A meta-analysis. Journal of American Medical Association, 288(6), 728-737.

Cardoso, V. C., Simões, V. M. F., Barbieri, M. A., Silva, A. A. M., Bettiol, H., Alves, M. T. S. S. B., \& Goldani, M. Z. (2007). Profile of three Brazilian birth cohort studies in Ribeirão Preto, SP and São Luís, MA. Brazilian Journal of Medical and Biological Research, 40(9), 1165-1176.

Casey, P. H., Whiteside-Mansell, L., Barret, K., Bradley, R. H., \& Gargus, R. (2006). Impact of prenatal and/or postnatal growth problems in low birth weight preterm infants on school-age outcomes: An 8-year longitudinal evaluation. Pediatrics, $118(3), 1078-1086$.

Conrad, A. L., Richman, L., Lindgren, S., \& Nopoulos, P. (2009). Biological and environmental predictors of behavioral sequelae in children born preterm. Pediatrics, 125, 83-89.
Dahl, L. B., Kaaresen, P. I., Tunby, J., Handegard, B. H., Kvernmo, S., \& Ronning, J. A. (2006). Emotional, behavioral, social, and academic outcomes in adolescents born with very low birth weight, Pediatrics, 118, 449-460.

Elgen, I., Sommerfelt, K., \& Ellertsen, B. (2003). Cognitive performance in a low birth weight cohort at 5 and 11 years of age. Pediatric Neurology, 29(2), 111-116.

Espírito Santo, J. L., Portuguez, M. W., \& Nunes, M. L. (2009). Cognitive and behavioral status of low birth weigth preterm children raised in a developing country at preschool age. Journal of Pediatrics, 85(1), 35-41.

Fleitlich, B., Córtazar, P. G., \& Goodman, R. (2000). Questionário de Capacidades e Dificuldades (SDQ). Infanto-Revista de Neuropsiquiatria da Infância e Adolescência, 8(1), 44-50.

Ferraz, T. R., \& Neves, E. T. (2011). Fatores de risco para baixo peso ao nascer em maternidades públicas: um estudo transversal. Revista Gaúcha de Enfermagem, 32(1), 86-92.

Gallo, E. A. G., Anselmi, L., Dumith, S. C., Scazufca, M., Menenzes, A. M. B., Hallal, P. C., \& Matijasevich, A. (2011). Tamanho ao nascer e problemas de saúde mental aos 11 anos em uma coorte brasileira de nascimentos. Cadernos de Saúde Pública, 27(8), 1622-1632.

Goodman, R. (1997). The Strengths and Difficulties Questionnaire: A research note. Journal of Child Psychology and Psychiatry, $38(5), 581-586$.

Goodman, R. (2001). Psychometric properties of Strengths and Difficulties Questionnaire. Journal of American Child Adolescent Psychiatric, 40(11), 1337-1345.

Hack, M., Youngstrom, E. A., Cartar, L., Schluchter, M., Taylor, H.G., Flannery, D., . . . Borawski, E. (2004). Behavioral outcomes and evidence of psychopathology among very low birth weight infants at age 20 years. Pediatrics, 114, 932-940.

Hayes, B., \& Sharif, F. (2009). Behavioural and emotional outcome of very low birth weight infants: Literature review. Journal of Maternal-Fetal and Neonatal Medicine, 22(10), 849-956.

Johnson, S. (2007). Cognitive and behavioural outcomes following very preterm birth. Seminars in Fetal and Neonatal Service, 12, 363-373.

Linhares, M. B. M., Bordin, M. B. M., \& Carvalho, A. E. V. (2004). Aspectos do desenvolvimento psicológico da criança ex-prematura na fase escolar. In E. Marturano, M. B. M. Linhares \& S. R. Loureiro (Eds.), Vulnerabilidade e proteção: indicadores na trajetória de desenvolvimento escolar (pp. 75106). São Paulo: Casa do Psicólogo.

Martins, I. M. B., Linhares, M. B. M., \& Martinez, F. E. (2005). Indicadores de desenvolvimento na fase pré-escolar de crianças nascidas pré-termo. Psicologia em Estudo, 10(2), 235-243.

Marturano, E. M., \& Loureiro, S. R. (2003). O desenvolvimento socioemocional e as queixas escolares. In A. Del Prette \& Z. A. Del Prette (Eds.), Habilidades sociais, desenvolvimento e aprendizagem. Questões conceituais, avaliação e intervenção (pp. 259-292). Campinas: Alínea.

Masten, A. S., \& Gewirtz, A. H. (2006). Vulnerability and Resilience in Early Child Development. In K. McCartney, D. Phillips (Eds.), Blackwell Handbook of Early Childhood Development (pp. 22-43). Oxford: Blackwell Publishing. 
Ment, L. R., Vohr, B., Allan, W., Katz, K. H., Schneider, K. H., Westerved, M., ... Makuch, R. W. (2003). Change in cognitive function over time in very low birth weight infants. Journal of American Medical Association, 289(6), 705-711.

Milligan, D. W. A. (2010). Outcomes of children born very preterm in Europe. Archives of Diseases Child Fetal Neonatal, 95(4), 234-240.

Msall, M. E., \& Park, J. J. (2008). The spectrum of behavioral outcomes after extreme prematurity: Regulatory, attention, social, and adaptive dimensions. Seminars of Perinatology, $32,42-50$.

Oliveira, C. G. T., Enumo, S. R. F., Azevedo Jr., R. R., \& Queiroz, S. S. (2011). Indicadores cognitivos, linguísticos, comportamentais e acadêmicos de pré-escolares nascidos prétermo e a termo. Psicologia: Teoria e Pesquisa, 27(3), 73-80.

Organização Mundial da Saúde - OMS (1994). CID-10: classificação estatística internacional de doenças e problemas relacionados à saúde. São Paulo: EDUSP.

Reijneveld, S. A., Kleine, M. J. K., van Baar, A. L., Kollée, L. A. A., Verhaak, C.M., Verhulst, F. C., \& Verloove-Vanhorick, S. P. (2006). Behavioural and emotional problems in very preterm and very low birthweight infants at age 5 years. Archives of Disease in Childhood. Fetal \& Neonatal Edition, 91, 423-428.

Rodrigues, M. C. C., Mello, R. R., \& Fonseca, S. C. (2006). Learning difficulties in schoolchildren born with very low birth weight. Jornal de Pediatria, 82(1), 6-14.

Sabet, F., Richter, L. M., Ramchandani, P. G., Stein, A., Quigley, M. A., \& Norris, S. A. (2009). Low birthweight and subsequent emotional and behavioural outcomes in 12-year-old children in Soweto, South Africa: Findings from birth to twenty. International Journal of Epidemiology, 38, 944-954.

Saigal, S. (2000). Follow up of very low birthweight babies to adolescence. Seminars in Neonatology, 5, 107-118.

Sameroff, A. J., \& Fiese, B. H. (2005). Models of development and developmental risk. In C. H. Zeanah Jr (Ed.), Handbook of infant mental health (2a ed., pp. 3-19). Nova Iorque: The Guilford Press.
Scott, M. N., Taylor, H. G., Fristad, M. A., Klein, N., Espy, K. A., Minich, N., \& Hack, M. (2012). Behavior disorders in extremely preterm/extremely low birth weight in kindergarten. Development Behavior Pediatric, 33, 202-213.

Silva, A. A., Barbieri, M. A., Cardoso, V. C., Batista, R. F., Simões, V. M., Vianna, E. O., . . Bettiol, H. (2011). Prevalence of non-communicable diseases in Brazilian children: Follow-up at school age of two Brazilian birth cohorts of the 1990's. BMC Public Health, 11, 486-518.

Sommerfelt, K., Andersson, H. W., Sonnander, K., Ahlsten, G., Ellertsen, B., Markestad, T., . . . Bakketeig, L. S. (2001). Behavior in term, small for gestational age preschoolers. Early Human Development, 65(2), 107-121.

Vanderbilt, D., \& Gleason, M. M. (2010) Mental health concerns of the premature infant through the lifespan. Child and Adolescent Psychiatric Clinics of North America, 19, 211-228.

Wiles, N. J., Peters, T. J., Heron, J., Gunnell, D., Emond, A., \& Lewis, G. (2006). Fetal growth and childhood behavioral problems: Results from the ALSPAC cohort. American Journal of Epidemiology, 163(9), 829-837.

Winchester, S. B., Sullivan, M. C., Marks, A. K., Doyle, T., DePalma, J., \& McGrath, M. M. (2009). Academic, social, and behavioral outcomes at age 12 of infants born preterm. Western Journal of Nursing Research, 31, 853-871.

Woerner, W., Fleitlich-Bilyk, B., Martinussen, R., Fletcher, J., Cucchiaro, G., Dalgalarrondo, P., .. Tannock, R. (2004). The Strengths and Difficulties Questionnaire overseas: Evaluations and applications of the SDQ beyond Europe. European Child \& Adolescent Psychiatry, 13(Suppl. 2), 1147-1154.

Zwicker, J. G., \& Harris, S. R. (2008). Quality of life of former preterm and very low birth weight infants from preschool-age to adulthood: A systematic review. Pediatrics, 121(2), 366-376. 